\title{
Giant magnetoimpedance (GMI) effect in soft melt-extracted magnetic amorphous fibres
}

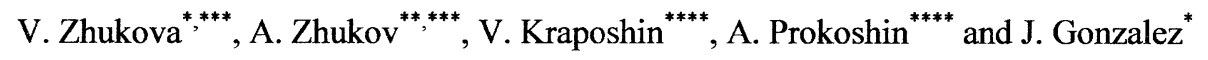 \\ *Department of Materials Physics, Faculty of Chemistry, P.O. Box 1072, 20080 San Sebastian, Spain \\ ** Instituto de Ciencia de Materiales, CSIC, 28049 Cantoblanco, Madrid, Spain \\ ***"TAMag Iberica" S.L., Avda. de Remedios 41-3a, Colmenar Viejo, Madrid, Spain. \\ ${ }^{* * *}$ I.P. Bardin State Science Center for Ferrous Metallurgy, Moscow Russia
}

$\mathrm{Co}_{79.6} \mathrm{Fe}_{4.6} \mathrm{Si}_{9.2} \mathrm{~B}_{2.7} \mathrm{Mo}_{3.9}$ amorphous fibres were produced by the modified melt-extraction process. Studied fibres exhibit GMI ratio of the order of $50 \%$ in as-cast state which is connected to their soft magnetic behaviour and small magnetostriction constant. Effect of ac current frequency and amplitude and current annealing (current intensity from 88 to $95 \mathrm{~mA}$, annealing time up to $28 \mathrm{~min}$ ) on GMI effect has been studied.

Key words: giant magnetoimpedance, soft magnetic fibres, hysteresis loops, current annealing

\section{Introduction}

Magnetoimpedance (MI) effect in amorphous and polycrystalline materials has been intensively studied in the last decade owing to their exciting technological applications. This MI effect is related with the change of the impedance experimented by a magnetic material under the effect of an external $d c$ magnetic field. In the last years a lot of works have been published dealing on MI from both theoretical and an experimental approach ${ }^{1)-5 \text { ). }}$ In addition, different geometries and materials have been tested and reported. It must be mentioned that in the case of amorphous ferromagnetic wires, exhibiting nearly-zero magnetostriction constant, several hundreds percent changes in impedance have been reported being in this case the effect called giant magnetoimpedance (GMI) Thus, relative changes of the electrical impedance upon $d c$ applied field of the order of $300 \%$ can be easily achieved in conventional amorphous wires (with diameter about $100 \mu \mathrm{m}$ ) with vanishing magnetostriction ${ }^{2)-4)}$. Although generally glass coated microwires exhibit a more reduced GMI effect ${ }^{6), 7)}$, significant progress has been recently achieved in tailoring of the glass-coated microwires with high GMI ratio (up to $600 \%{ }^{8}$ ).

The GMI effect has been interpreted in terms of the classical electrodynamics considering the change of the penetration depth of the $a c$ current in a magnetic conductor caused by the $d c$ magnetic applied field. The frequency, $f$, of the $a c$ current flowing along the sample must be high enough (typically above $100 \mathrm{kHz}$ ). The electrical impedance, $Z$, of a magnetic conductor is given by ${ }^{2), 3)}$

$$
Z=R_{\mathrm{dc}}(k r) J_{\mathrm{o}}(k r) / 2 J_{1}(k r)
$$

with $k=(1+j) / \delta \quad$ where $J_{\mathrm{o}}$ and $J_{1}$ are the Bessel functions and $\delta$ the penetration depth given by

$$
\delta=\left(\pi \sigma \mu_{\phi} f\right)^{-1 / 2}
$$

where $\sigma$ is the electrical conductivity, $f$ is the frequency of the current along the sample, and $\mu_{\phi}$ is the circular permeability assumed to be scalar. The $d c$ applied magnetic field, $H$, changes the penetration depth through the modification of $\mu_{\phi}$ which finally results in a change of the impedance ${ }^{2)-4}$. Such enhanced GMI ratio was explained by the particular domain structure of the nearlyzero magnetostrictive amorphous wires consisting of an outer domain sheath with high circumferential permeability and outstanding magnetic softness ${ }^{2)}, 3$ ).

On the other hand, an alternative family of tiny wires fine amorphous magnetic fibres produced by extraction method is known since 80-th. These amorphous magnetic fibres made by the melt-extraction process ${ }^{9)-11)}$ posses good soft magnetic properties with low $d c$ coercivities (about few $\mathrm{Am}^{-1}$ ) and high enough magnetic permeability 11). Their magnetization process and GMI at relatively low $(\mathrm{MHz})$ frequencies are still poorly studied.

A main advantage of this process, as comparing with others is that it allows to obtain fibres when extracting with a wide range of metals and oxide ceramics. The present paper is dealing on the GMI effect of fine amorphous magnetic fibres with nearly-zero magnetostriction constant, fabricated by modified meltextraction process.

\section{Experimental technique}

$\mathrm{Co}_{79.6} \mathrm{Fe}_{4.6} \mathrm{Si}_{9.2} \mathrm{~B}_{2.7} \mathrm{Mo}_{3.9}$ amorphous fibres were obtained by modified melt-extraction technique initially developed by Maringer and Mobley ${ }^{9)}$ and later modified by Rudkowski et al. 10), 11). This technique consists of melting the metallic ingot of few grams by a clean heat source such as rf induction or CW infrared and extracting the fibre from the melt by means of a rapidly rotating sharpened wheel (made from a refractory metal such as molybdenum) moving at tangential speeds between 10 and $50 \mathrm{~ms}^{-1}{ }^{12}$ ). The process produces fibres typically 10 $\mathrm{m}$ in length and 30-60 $\mu \mathrm{m}$ in diameter fibres. 


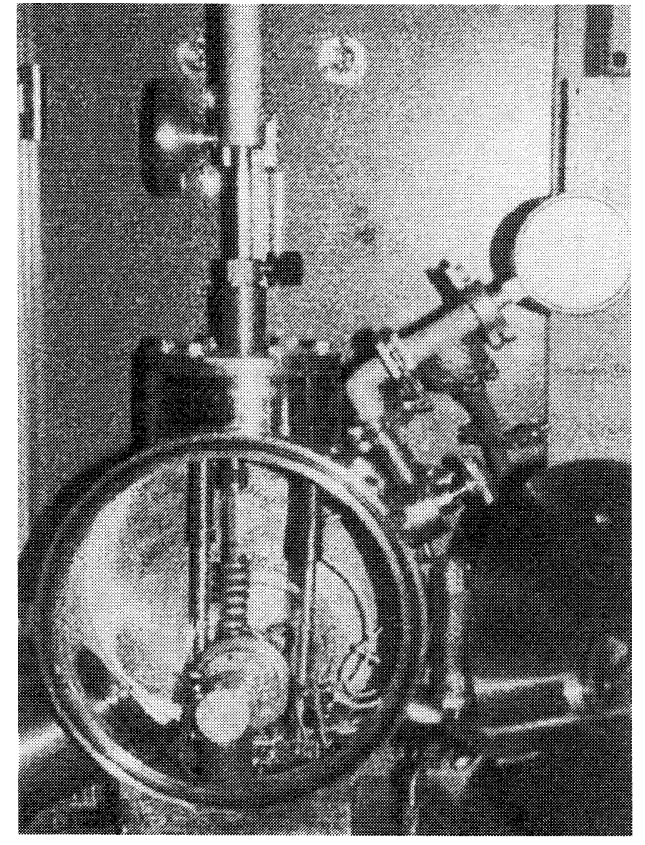

Fig.1. Experimental set-up for fabrication of the fibers.

We used a modified set-up for the fabrication of fibres by melt-extraction, which was assembled with the quenching block placed in the interior of a vacuum chamber. The other difference with the experimental setup described in 10)-12) is that the molten alloy is supplied from the top of the quenching wheel. The quenching block consists mainly of: 1) an inductor with the quartz ampoule inside having a round orifice and served as the crucible for the alloy melting; 2) quenching disc made out of a refractory metal and placed on the shaft of the micromotor; 3) a mechanism for the vertical displacement of the quartz ampoule. The linear velocity of the disc edge was about $30 \mathrm{~m} / \mathrm{s}$. An overview of the set-up is shown in Fig. 1.

This set-up allows to obtain thin few meters long fibre with the diameter of 30-60 $\mu \mathrm{m}$.

Samples $7 \mathrm{~cm}$ in length and $50 \mu \mathrm{m}$ in diameter have been used for our experimental studies. The fibres have been submitted to the current annealing in air in two steps: the first one at $88 \mathrm{~mA}$ for 1-28 minutes of evaluated by means of the four-point technique. The ac electrical current flowing along the metallic nucleus duration, followed by a second one at $95 \mathrm{~mA}$ for 2 minutes.

The electrical impedance of the microwire was was of a frequency $(f)$ ranging between 1 and $10 \mathrm{MHz}$ and amplitude $(I)$ between 1 and $2 \mathrm{~mA}$. The magnetoimpedance ratio, $\Delta Z / Z$, has been defined as:

$$
\Delta Z / Z=\left[Z(H)-Z\left(H_{\max }\right)\right] / Z\left(H_{\max }\right)
$$

where $d c$ longitudinal magnetic field, up to $H_{\max }=2400$ $\mathrm{A} / \mathrm{m}$ was supplied by a long solenoid.

\section{Experimental results}

Studied fibres show soft magnetic behaviour (see their hysteresis loops in Fig.2). The value of coercive field, $H_{c}$

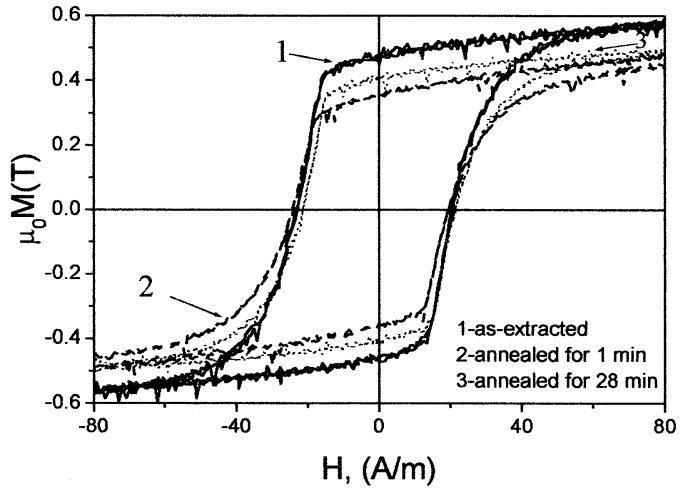

Fig.2. Hysteresis loops of as-extracted and current annealed fibers ( 1 -as-extracted, 2 -annealed at $I=88 \mathrm{~mA}$ for $1 \mathrm{~min}$ and 3 annealed at $I=88 \mathrm{~mA}$, for $28 \mathrm{~min}$.).

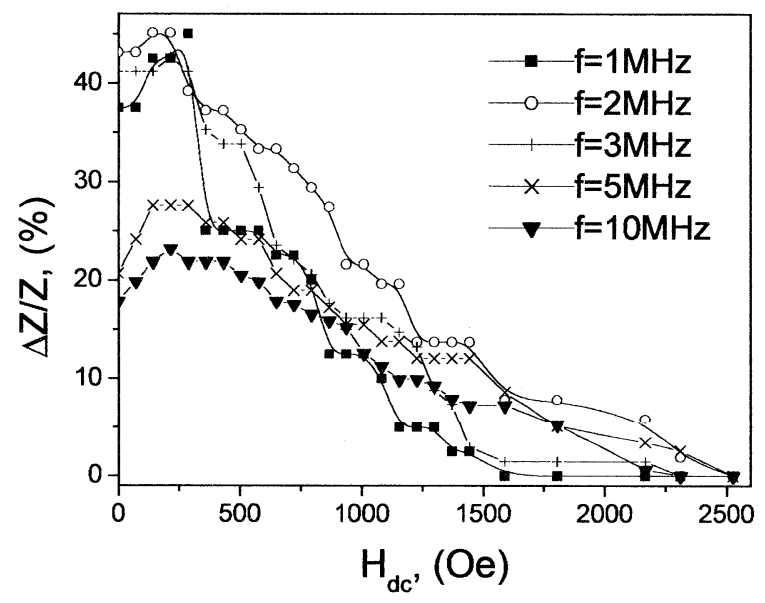

Fig.3 . $\Delta Z / Z(H)$ dependencies measured in as-extracted fiber for driving current amplitude $I=1 m A$ with the frequency, $f$, as a parameter.

$=20 \mathrm{Am}^{-1}$, is similar to that of microwires obtained by Taylor-Ulitovsky method ${ }^{8)}$. Current annealing slightly modify the hysteresis loop : the remanent magnetization, $\mu_{0} M_{r}$, slightly decreases even after short $(1 \mathrm{~min})$ current annealing, while $H_{c}$ is almost insensitive to the heat treatment. On the other hand the magnetostriction constant measured by the SAMR method shows values of about $10^{-6}$.

$\Delta Z / Z(H)$ dependences measured at different frequencies, $f$, of the $a c$ driving current with the amplitude, $I=1 \mathrm{~mA}$, for as-extracted sample are presented in the Fig. 3. At all frequencies GMI shows a slight maximum at certain $d c$ axial magnetic field, $H_{\mathrm{m}}$, around $200 \mathrm{~A} / \mathrm{m}$, which is almost independent on the frequency. At $f>2 \mathrm{MHz}$ the maximum GMI ratio, $(\Delta Z / Z)_{\max }$, decreases drastically with the frequency, which is attributed to the reduction of circular permeability.

The $\Delta Z / Z(H)$ dependences measured at frequency of 1 and $10 \mathrm{MHz}$ with a value of the $a c$ driving current amplitude, $I$, of $2 \mathrm{~mA}$ are presented in Fig. 4 for the asextracted and treated samples. A remarkable effect of the current annealing can be attributed to the stress relaxation. 


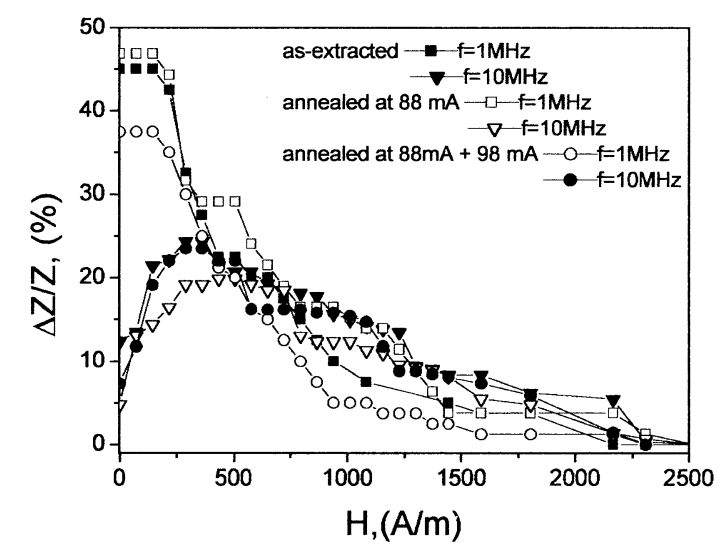

Fig.4. $\triangle Z / Z(H)$ dependencies measured in as-extracted sample and annealed at $88 \mathrm{~mA}$ for 1 minute and at 88 $\mathrm{mA}$ for 1 minute $+95 \mathrm{~mA}$ for 2 minutes at $I=2 \mathrm{~mA}$ and $f=1 \mathrm{MHz}$ and $f=10 \mathrm{MHz}$.

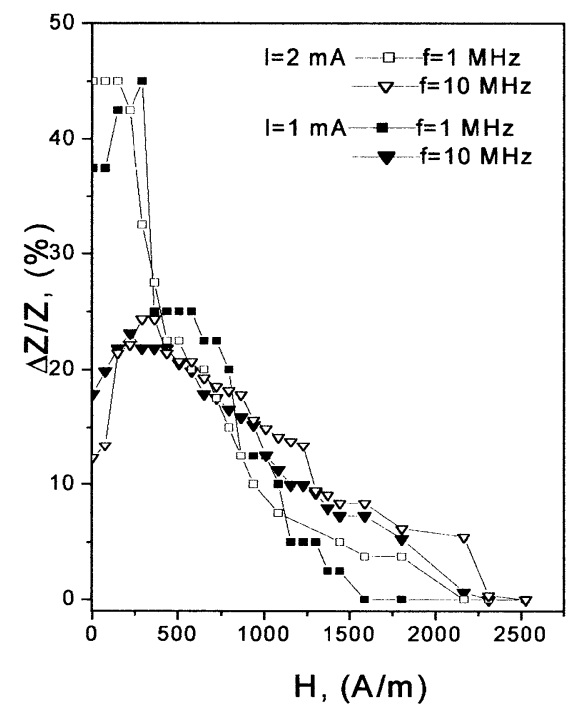

Fig.5. $\triangle Z / Z(H)$ dependencies measured in as-extracted sample at frequencies $f=1 \mathrm{MHz}$ and $f=10 \mathrm{MHz}$ with current amplitude as a parameter.

The $\Delta Z / Z(H)$ dependencies measured $f=1$ and $10 \mathrm{MHz}$ with $I$ as a parameter $(I=1$ and $2 \mathrm{~mA}$ ) has been plotted in Fig 5. Observed difference is related with the circular magnetization process (i.e. with the effect of a circular magnetic field, produced by an $a c$ driving current, on the circumferential magnetic permeability). This effect was previously observed and described by various authors ${ }^{6}$ ), 13), 14). In fact, the driving current dependence of the GMI denotes the non-linear character of GMI effect, i.e. that the domain structure of the outer shell plays an important role ${ }^{2)}$. It is expected that, as in case of the axial hysteresis loop, the increasing of the magnetic field amplitude results in an increase of the circular coercivity ${ }^{14)}$.

Fig. 6 shows the $\triangle Z / Z(H)$ dependency of the annealed fibre measured at $f=3 \mathrm{MHz}$ (Fig. 6a) and $f=10 \mathrm{MHz}$ (Fig $6 b)$ for both positive and negative magnetic fields. The MI response presents clear asymmetry for the both frequencies, showing two peaks at low magnetic fields. Such asymmetrical character with two peaks of the MI
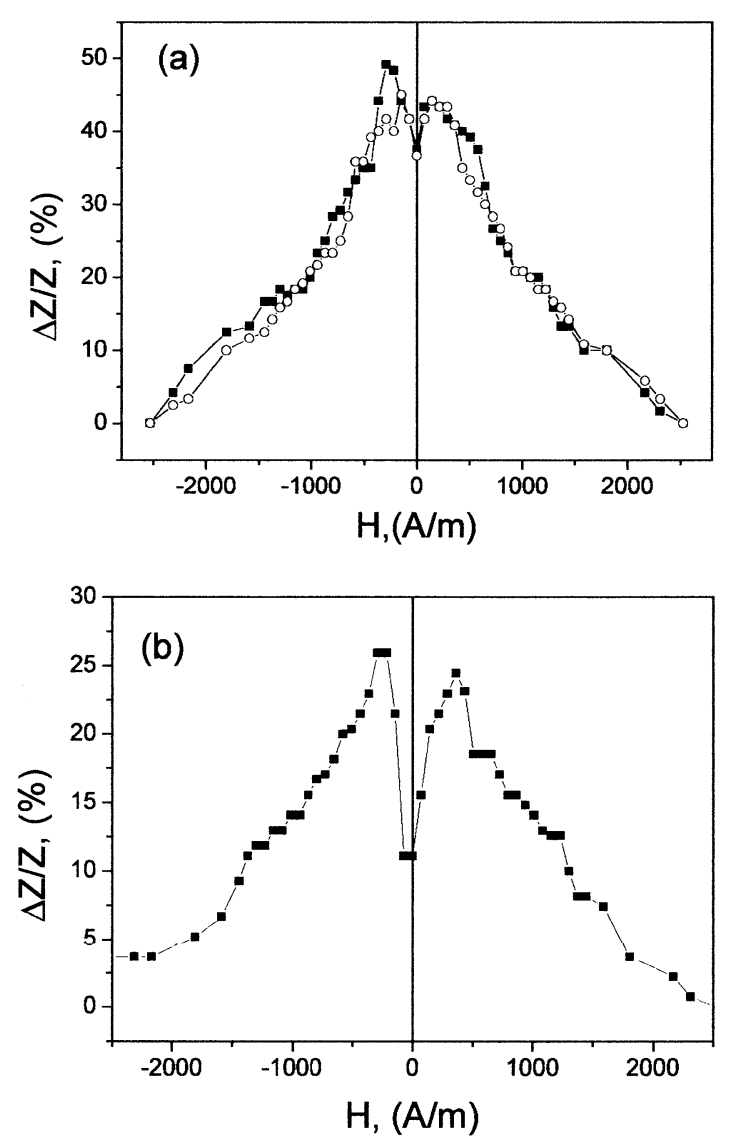

Fig.6. $\triangle Z / Z(H)$ dependencies measured in annealed at 88 $\mathrm{mA}$ for 1 minute $+95 \mathrm{~mA}$ for 2 minutes sample measured at $3 \mathrm{MHz}$ (a) and $10 \mathrm{MHz}$ (b).

curves has been recently found by Ciureanu et al. ${ }^{16)}$ in a $\mathrm{CoFeSiNNb}$ melt-extracted fiber.

The sensitivity of about $3 \% / \mathrm{Am}^{-1}$ has been observed at low magnetic $d c$ field, which is quite similar to that reported in 16). (We define the sensitivity as the ratio of the relative change in sample impedance to the applied magnetic field: $S=(\Delta Z / Z) / H_{\text {lin }}$, where $H_{\text {lin }}$ is the $d c$ field range over which $\Delta Z / Z$ is linear.)

\section{Discussion}

The significant GMI ratio should be attributed to relatively low magnetic anisotropy field, that permits to achieve an elevated magnetic permeability of the sample. In fact, many experimental and theoretical results 11,8$), 17)$ ${ }^{19)}$ pointed out that the low magnetic anisotropy is directly related with the GMI effect. For instance, it was theoretically shown in 1) and 20) that the axial dependence of the GMI spectra is mainly determined by the type of magnetic anisotropy. It was shown particularly, that the circumferential anisotropy leads to the observation of the maximum of the real component of wire impedance (and consequently of the GMI ratio) as a function of the external magnetic field. In contrast, in the case of axial magnetic anisotropy the maximum value of the GMI ratio 
corresponds to the zero magnetic field ${ }^{18)}$, i.e. $(\Delta Z / Z)(H)$ has a shape of monotonous decay. Consequently, a nondiagonal components of the magnetic permeability tensor and impedance tensor were introduced in 13), 18) -20) in order to describe such circumferential anisotropy. Therefore, for the samples with well-defined maximum on the axial field dependence of GMI ratio, an importance of the non-diagonal components of the permeability tensor should be underlined.

As has been shown, the observed GMI response presents an asymmetrical character, with two peaks of different amplitude, with the peak at positive field smaller than that at negative field when the response is plotted after a positive longitudinal saturation of the microwire. Such behaviour is different from that observed by P.Ciureanu et al ${ }^{16)}$, where only single peak GMI effect has been observed in as-extracted CoFeSiNNb fiber. To explain this behaviour in 21) was assume that this strong asymmetrical double-peak MI response of the fibre with unequal peak amplitude, is the result of the dispersion of the helical magnetic anisotropy induced in this material by the complex distribution of internal stresses.

This GMI response may be viewed as the equivalent of the response reported by Blanco et al. ${ }^{22)}{ }^{23)}$, Panina and colleagues ${ }^{24)}$ for a Co-rich Unitika wire with helical anisotropy induced through torsion annealing ${ }^{23)}$ or twisting ${ }^{22), 25)}$ biased by a circumferential $d c$ field, playing the helical anisotropy of magnetoelastic character or that developed by the torsion annealing an important role to explain these effects.

It has been widely accepted that the amorphous wires with vanishing negative magnetostriction exhibit a peculiar domain structure consisting of an internal axially magnetized core and an outer shell with the magnetization circularly oriented. This domain structure is originated from the magnetoelastic anisotropy associated to the internal stresses resulting to be very favorable for high GMI effect. It was theoretically and experimentally demonstrated that tensile and torsion stresses modify this domain structure changing so the spatial magnetization distribution close to the surface ${ }^{22)}$.

\section{Conclusions}

Magnetically soft $\mathrm{Co}_{79.6} \mathrm{Fe}_{4.6} \mathrm{Si}_{9.2} \mathrm{~B}_{2.7} \mathrm{Mo}_{3.9}$ amorphous fibres have been obtained by modified melt-extraction technique. Such samples present considerable (50\%) GMI ratio. Substantial dependence of $(\Delta Z / Z)(H)$ on frequency and amplitude of the $A C$ driving current bias current has been observed. Asymmetrical character $(\Delta Z / Z)(H)$ is related to the helical magnetic anisotropy. Current annealing modifies slightly both magnetic properties and GMI effect.

\section{Acknowledgement}

This work has been supported by the Spanish Science Council, Project MAT-2000-1468-C02-02 and
MAT-2001-0082-C04-04.

\section{References}

1) J.M. Barndiarán, A. García-Arribas, L. Muñoz and G.V. Kurlyandskaya, IEEE Trans.Magn., 38, 3051 (2002)

2) L.V. Panina and K. Mohri, Appl.Phys.Lett., 65, 1189 (1994)

3) R. S. Beach and A. E. Berkowicz, Appl.Phys.Lett., 64, 3652 (1994)

4) F.L. Machado, C. S. Martins and B.M. Razebde, Phys. Rev. B, 51, 3926 (1995)

5) D. Ménard, M. Britel, P. Ciureanu and A. Yelon, J. Appl. Phys. 84, 2805 (1998).

6) M. Vázquez, A. Zhukov, P. Aragoneses, J. Arcas, J.M. Garcia-Beneytez, P. Marin and A. Hernando, IEEE Trans.Magn., 34, 724 (1998)

7) A.F. Cobeño, A. Zhukov, J.M. Blanco and J. González, J.Magn.Magn.Maert., 234, L359 (2001)

8) A. Zhukov, J. Magn and Magn, Mater. 242-245 216 (2002).

9) E. Maringer and C.E. Mobley, U.S. Patent No. 3871439 (March, 1975)

10) P. Rudkowski, G. Rudkowska and J.O. Strom-Olsen, Mater.Sci.Eng.A,, 133, 158 (1991)

11) P. Rudkowski, G. Rudkowska, J.O. Strom-Olsen, C. Zeler and R. Cordery, J.Appl.Phys., 69, 5017 (1991)

12) P. Rudkowski, J.O. Strom-Olsen, G. Rudkowska, A. Zaluska and P. Ciureanu, IEEE Trans.Magn., 31, 1224 (1995).

13) P. Aragoneses, A. Zhukov, J. González, J.M. Blanco and L. Domínguez, Sensors and Actuators A, 81/1-3, 86 (2000).

14). K.V. Rao, F.B. Humphrey and J.L. Costa Krämer, J.Appl.Phys., 76, 6204 (1994)

15). A.P. Zhukov, B.K. Ponomarev, Sov. Phys. Solid State, 31 26 (1989).

16) P. Ciureanu, I. Khalil, L.G.C. Melo, P. Rudkowski and A. Yelon, J.Magn.Magn.Mat., 249, 305 (2002)

17) J.P. Sinnecker, E.H.C.P. Sinnecker, A. Zhukov, J.M. GarciaBeneytez, M.J. Garcia Prieto and M. Vázquez, J. Phys. IV France 8, Pr2-225 (1998)

18). N.A. Usov, A.S. Antonov and A.N. Lagar'kov, J.Magn.Magn.Mat., 185, 159 (1998)

19) A.S. Antonov and I.T. Iakubov, J.Phys.D: Appl.Phys., 32, 1204 (1999)

20) D.P.Makhnovskiy, L. V. Panina, and D.J.Mapps, Phys. Rev. B 63, 144424 (2001).

21) D. Atkinson and P. Squire, IEEE Trans.Magn., 33, 3364 (1997)

22) J.M. Blanco, A. Zhukov and J. González, J.Phys.D. Appl.Phys., 32, 3140 (1999)

23) J.M. Blanco, A. Zhukov and J. González, J.Phys.D: Appl.Phys., 34, L31 (2001)

24) L.V. Panina, K. Mohri and D. Makhnovskiy, J.Appl.Phys., 85, 5444 (1999)

25) K. Mohri, U.S. Patent 5, 994,899 (1996)

( Received May 8, 2003; Accepted October 29, 2003 ) 